\title{
Esquemas urbano-forestales en una «ciudad oasis» de zona árida: Mendoza (Argentina). Influencia sobre el consumo energético residencial
}

\section{Urban-forest configurations in an "oasis city" of arid zone: Mendoza (Argentina). Influence on residential energy consumption}

M. $^{\text {a A. Ruiz }}{ }^{(*)}$, E. N. Correa ${ }^{(*)}$

\section{RESUMEN}

La ciudad de Mendoza (Argentina) se caracteriza por un intenso bosque urbano, calificando como una «ciudad oasis» inserta en una zona árida. Sin embargo, la isla de calor generada y sus consecuencias energético-ambientales enfatizan la necesidad de replantear el modo de forestar la ciudad. Por tanto, el objetivo del trabajo es evaluar el efecto de distintos esquemas urbanos-forestales sobre el consumo de energía residencial. Para ello, se monitoreó micro-climáticamente nueve canales viales representativos en invierno y verano y se simuló el comportamiento térmico de un prototipo de vivienda inserto en cada canal vial. Los resultados indican que los diseños urbanos que controlan la radiación solar mediante una adecuada distribución de la sombra y permiten una mejor circulación del viento, minimizan los consumos de energía auxiliar hasta un 30\%. Esto permite generar propuestas técnicas factibles de incorporar en códigos urbanos y, como consecuencia, un uso más eficiente del arbolado en alineación.

Palabras clave: Consumo energético residencial; canales viales urbanos forestados; ciudad oasis; zona árida.

\section{ABSTRACT}

The Mendoza Metropolitan Area (Argentina) is characterized by an intense urban forest, which makes it an "oasis city" inserted in an arid area. However, the generated heat island and their energy-environmental consequences emphasize the need to rethink the way of tree plantation in the city. Therefore, the goal of this study was to evaluate the effect of different urban-forest schemes on residential energy consumption. For that purpose, nine representative urban canyons were micro-climatically monitored in winter and summer and the thermal behavior of a prototype housing inserted in each urban canyon was simulated. The results indicate that the urban designs that control the solar radiation by means of an adequate distribution of the shade and that allow a better wind circulation, minimize the auxiliary energy consumption up to $30 \%$. This allows generating feasible technical proposals to incorporate into urban codes and therefore, more efficient use of trees lined.

Keywords: Residential energy consumption; forested urban canyons; oasis city; arid zone.

(*) Instituto de Ambiente, Hábitat y Energía (INAHE) - CCT CONICET Mendoza

Persona de contacto/Corresponding author: aruiz@mendoza-conicet.gob.ar (M. ${ }^{\text {a }}$ A. Ruiz)

ORCID: http://orcid.org/oooo-0002-7336-082X. (M. ${ }^{a}$ A. Ruiz); http://orcid.org/oooo-0003-1690-076X. (E. N. Correa).

Cómo citar este artículo/Citation: M. a A. Ruiz, E. N. Correa. (2018). Esquemas urbano-forestales en una «ciudad oasis» de zona árida: Mendoza (Argentina). Influencia sobre el consumo energético residencial. Informes de la Construcción, 70(549): e239. https://doi. org/10.3989/ic.16.135

Copyright: (C) 2018 CSIC. Este es un artículo de acceso abierto distribuido bajo los términos de la licencia de uso y distribución Creative Commons Reconocimiento 4.0 Internacional (CC BY 4.0). 


\section{INTRODUCCIÓN}

Las ciudades tienen una acción transformadora del espacio natural sobre el que se asientan alterando en mayor o menor medida los parámetros de sus climas locales. Uno de los principales cambios que causa la ciudad sobre el clima es el incremento de la temperatura del aire y el desarrollo de una isla de calor, que se traduce en una mayor temperatura del centro urbano en relación con el espacio rural circundante aire (1) (2) (3). Este fenómeno afecta el consumo residencial de energía, la calidad del aire y el grado de confort térmico en los espacios abiertos, entre otros. Sus efectos sobre el consumo de energía y el clima urbano, en particular la demanda de combustibles fósiles, se han abordado en diversos estudios (4) (5) (6). Para las urbes de más de 100.000 habitantes, el consumo de energía durante las horas pico se eleva entre 1,5 y 2,0\% por cada grado de aumento de la temperatura de la ciudad (7). Particularmente en la Argentina, la demanda residencial de energía eléctrica representa más del 40\% de la demanda total del distribuidor y el consumo aumenta de manera sostenida desde el año 2002. Paralelamente, la instalación de equipos de aire acondicionado en el país ha crecido 100\% en el periodo 2005-2009 (8).

En el Área Metropolitana de Mendoza (AMM), ciudad oasis de zona árida, la isla de calor urbana (ICU) ha sido estudiada en profundidad por Correa (9). Se verificó que la ICU se presenta en todas las estaciones del año, con intensidades máximas que oscilan entre los 8 y los $10^{\circ} \mathrm{C}$. En especial, se detectó que la ocurrencia de las máximas temperaturas se presenta generalmente durante el período de enfriamiento nocturno.

Una de las principales estrategias de mitigación de la ICU es la incorporación de la vegetación en la ciudad. La influencia de zonas verdes en el clima urbano ha sido ampliamente demostrada en la literatura internacional. Autores como Robinette (10), Yilmaz et al. (11) y Morakinyo et al. (12), entre otros, reconocen el papel de los árboles, la vegetación y el agua alrededor de los edificios en la determinación de las condiciones térmicas de los mismos. Asimismo, Correa et al. (13), Puliafito et al. (14) y Ruiz et al. (15) han estudiado la interacción de los parques y calles arboladas en el ambiente de Mendoza.

En particular, las autoras han evaluado el confort térmico en la baja densidad edilicia del AMM (16). Los resultados determinaron que la clave para maximizar la habitabilidad de los espacios es la adecuada combinación entre trama urbana, especie forestal y densidad edilicia. Otro aspecto importante que deriva del análisis, es la dependencia del modelo de ciudad respecto de la trama forestal, mostrando el alto grado de disconformidad térmica asociado al esquema sin forestación. Es importante destacar que el confort térmico está asociado al grado de habitabilidad de los espacios; pero además es necesario que el diseño de los espacios abiertos concilie buenas posibilidades de ventilación y enfriamiento de la ciudad, factores que condicionan la calidad del aire y el incremento de los consumos energéticos. Esto pone de manifiesto la necesidad de una planificación y manejo racional del recurso forestal de la ciudad como estrategia ineludible para su sustentabilidad.

De ello se desprende que es necesario analizar con profundidad el impacto de la combinación de distintas estructuras forestales, edilicias y urbanas para la concreción de la susten- tabilidad urbana en lo referente al consumo de energía de los espacios construidos.

Este trabajo busca analizar el nivel de consumo energético residencial asociado a distintos esquemas de canales urbanos forestados. El objetivo final es seleccionar las alternativas morfológicas óptimas en los aspectos energéticos y ambientales de los espacios abiertos en ciudades oasis de clima árido.

\section{METODOLOGÍA}

Con el fin de estudiar el impacto de la vegetación urbana sobre el consumo energético residencial, se ha monitoreado la situación microclimática de nueve canales viales urbanos durante los veranos 2009 y 2010 y el invierno 2010.

A continuación se detalla: (a) la selección de los casos de estudio; (b) la descripción del prototipo de vivienda; (c) la simulación del comportamiento térmico interior del prototipo de vivienda y (d) el método de monitoreo y recopilación de datos.

\subsection{Selección de los casos de estudio}

Este estudio ha sido llevado a cabo en el Área Metropolitana de Mendoza, en el centro-oeste de Argentina ( $32^{\circ} 40^{\prime}$ Latitud Sur, $68^{\circ} 51^{\prime}$ Longitud Oeste; 750 metros sobre el nivel del mar). Es la quinta ciudad más grande del país con 168 km2 y 1.055.679 habitantes (8).

Según la clasificación de Köppen-Greiger el clima de la cuidad es árido: BWh o BWk dependiendo de la isoterma usada (17). Está caracterizada por inviernos fríos (temperatura media del mes de julio: $7,3^{\circ} \mathrm{C}$ ) y veranos calurosos (temperatura media de enero: $24,9^{\circ} \mathrm{C}$ ), con importantes amplitudes térmicas diarias y estacionales. Los vientos son moderados y poco frecuentes (velocidad promedio: $11 \mathrm{Km} / \mathrm{h}$ ), la cantidad e intensidad de radiación solar es elevada (radiación solar incidente anual: $190 \mathrm{MJ} / \mathrm{m} 2$ ) y la precipitación media anual es de $198 \mathrm{~mm}$, con una concentración del 76 \% entre octubre y marzo (18).

La ciudad de Mendoza responde al modelo urbano abierto, es decir que presenta una trama urbana con calles anchas y construcciones relativamente bajas, donde la construcción de la sombra se basa en una intensa forestación dispuesta en paralelo a calles y avenidas. Además la vegetación se ve reforzada en parques, plazas y otros espacios abiertos. El desarrollo de la trama forestal ha permitido túneles "verdes» que dan la característica de un auténtico bosque dentro de la ciudad. Estas cualidades han sido reconocidas a nivel local e internacional y han merecido la calificación de la ciudad como «ciudad oasis» (19) (20) (21). En particular, el 84\% de las especies forestales del AMM corresponden a la primera y segunda magnitud y están representadas mayoritariamente por Platanus hispanica o plátano (22\%), Morus alba o morera (38\%) y Fraxinus excelsior o fresno europeo (19\%) (22).

Por otro lado, la estructura espacial del AMM se ha desarrollado en forma piramidal concentrando la mayor masa edilicia y en altura en el sector fundacional que corresponde al microcentro de la ciudad, la cual decrece progresivamente en altura y densidad hacia la periferia de la mancha urbana del AMM, hasta alcanzar las mínimas densidades en las áreas residenciales. En este aspecto, el espacio urbano se estruc- 
tura en dos densidades edilicias características: alta y baja. Actualmente el 80\% de la edificación del AMM corresponde a baja densidad (la relación entre el área total construida y el área total de superficie edificable es de 2,75) (16). Por otro lado, estudios previos del comportamiento de las temperaturas urbanas en relación con su entorno edilicio-vegetado (22) (23) (24) (25) revelan que la influencia de la vegetación sobre la condición microclimática del entorno se aprecia con mayor contundencia en el sector de baja densidad edilicia.

La mayoría de los cañones viales urbanos (CVU) de la zona de baja densidad coinciden con el uso residencial del suelo, a diferencia de lo que sucede en la alta densidad edilicia, donde aparece en gran medida el uso comercial. Según Sosa et al. (26), en las zonas residenciales del AMM las manzanas cuadradas sólo representan el 9\%, mientras que las rectangulares son el $54 \%$ de la trama urbana. Entre estas, existe una leve predominancia de las manzanas orientadas E-O (52\%). En el actual tejido del AMM, los cañones viales urbanos de 16, 20 y $30 \mathrm{~m}$ de ancho representan el $77 \%$ de la trama urbana residencial del AMM (27).

De acuerdo a lo discutido previamente, este estudio evalúa el impacto de distintas morfologías del entorno urbano forestado de baja densidad edilicia sobre los consumos energéticos de viviendas ubicadas en Área Metropolitana de Mendoza. Se consideraron los casos resultantes de la combinación de los tres anchos de canal vial predominantes $(16,20$ y $30 \mathrm{~m})$ y las tres especies forestales representativas (plátano, morera y fresno). Todos los canales viales evaluados están orientados Este-Oeste. Esta condición se estableció debido a dos factores: a) esta orientación presenta las mayores ganancias solares en la trama urbana del AMM, siendo las fachadas que miran al Norte las que reciben radiación directa durante mayor parte del día (28) (29); b) existe mayor porcentaje de viviendas en la orientación E-O que en la N-S.

En la Figura 1 se presentan fotografías hemiesféricas de los nueve casos de estudio y el recorrido solar durante los días estudiados en verano.

\subsection{Descripción del prototipo de vivienda}

Con el fin de determinar la influencia de la vegetación en los consumos de energía residenciales se ha elegido un prototipo de vivienda unifamiliar que es insertado por medio de simulación en cada uno de los nueve casos evaluados.

Se ha escogido una vivienda unifamiliar ya que el parque edilicio actual en el Área Metropolitana de Mendoza está constituido en su mayor parte por este tipo de edificaciones. En cuanto a la tipología seleccionada, se trata de la Casa o Chalet Neo-Colonial que predomina en el 44\% del AMM (30).

Esta tipología de chalet tiene la característica de ser compacta y su relación con el corazón de manzana es bastante pobre. Sin embargo, incorpora elementos tales como galerías, porches y atrios. En general, estos componentes son considera-

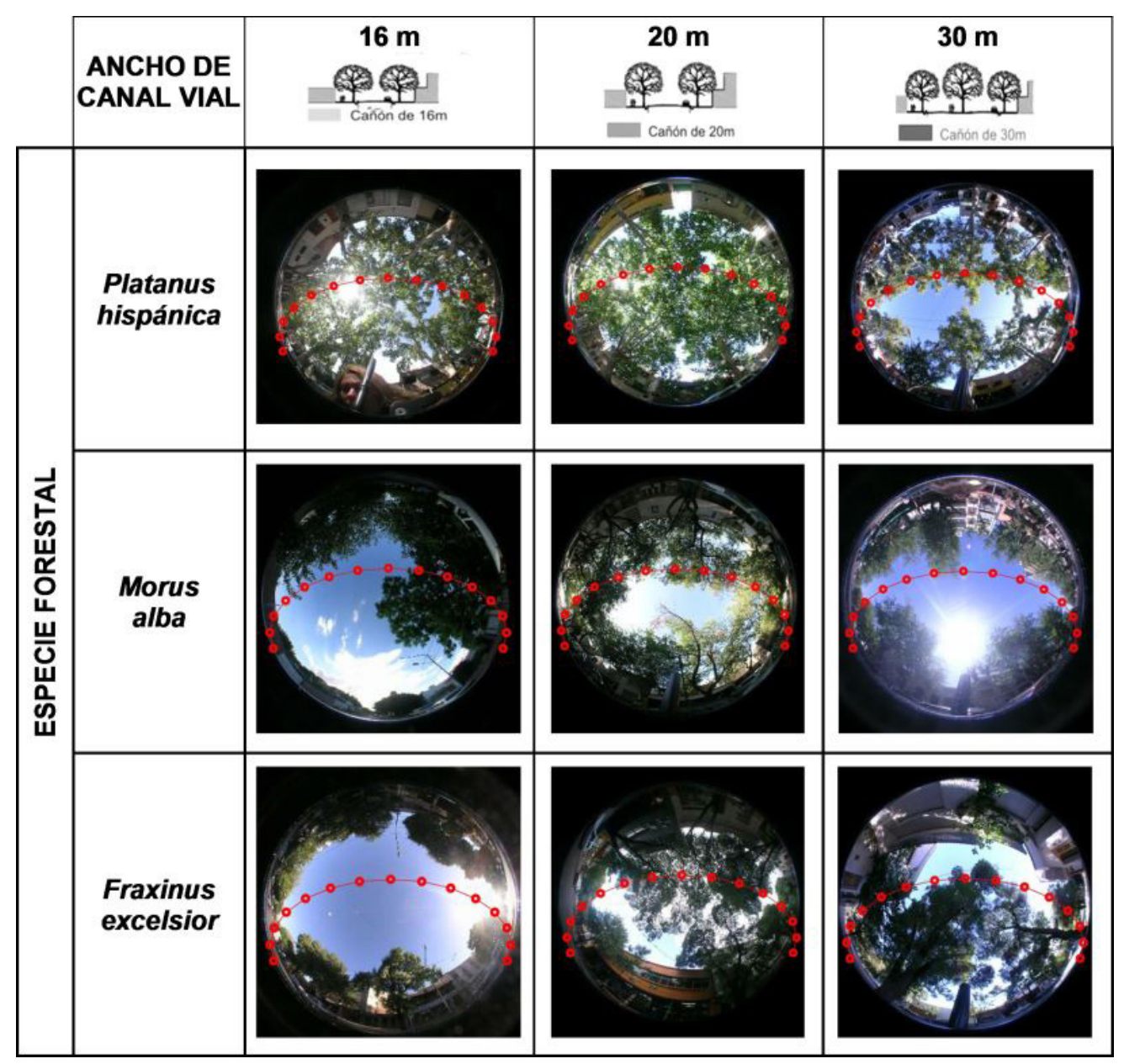

Figura 1. Recorrido solar de los nueve casos de estudio durante los días estudiados en verano. 
dos sólo como decorativos, a pesar de tener el potencial uso como parte fundamental de la envolvente para la regulación climática

La Figura 2 muestra la vivienda, con una superficie cubierta de $106 \mathrm{~m}^{2}$ y un volumen de $298,75 \mathrm{~m}^{3}$. La envolvente vertical, del tipo macizo, es de ladrillón revocado en ambas caras de $0,20 \mathrm{~m}$ de espesor (sin aislación). La cubierta, de tipo liviano, es inclinada, a dos aguas conformada por las siguientes capas: correas de madera y machimbre (Pinus elliotti), aislación térmica (lana de vidrio) y teja cerámica tipo colonial. En el espacio interior, la vivienda presenta cielorrasos suspendidos de yeso conformando una cámara de aire estanco que actúa de fuelle entre el exterior y el interior. El prototipo de vivienda consta de 8 locales o habitaciones, entre los cuales el Local 5 es el que presenta mayor vulnerabilidad respecto de las condiciones externas ya que es el único ambiente con ganancia solar directa al Norte. Además, este local funciona como estar-comedor, habitación que interesa especialmente por su intenso uso familiar. Por estas razones se ha decidido enfocar el estudio energético sobre este local. El área colectora total corresponde sólo a un $6 \%$ de la envolvente.

\subsection{Simulación del comportamiento térmico interior del prototipo de vivienda}

En la actualidad, se dispone de una gran variedad de programas para simulación energética de edificios. García-Alvarado et al. (31) hicieron una revisión de una docena de sistemas para simulación energética de edificios y sugieren que la selección del software apropiado para el análisis energético de residencias unifamiliares depende de la fase y extensión de su utilización, y por ende, la implementación requerida, la duración del estudio y características analizadas.

Con el propósito de simular la temperatura interna de la vivienda ubicada en cada canal vial urbano, en este trabajo se utilizó el programa SIMEDIF para Windows. Este software ha sido desarrollado en el INENCO (Instituto de Investigación en Energía No Convencional, Salta, Argentina) como una herramienta de diseño y simulación del comportamiento térmico transitorio de edificios con acondicionamiento natural, ganancias internas variables, enfriadores evaporativos e intercambiadores de calor aire-tierra (32). SIMEDIF ha sido empleado por numerosos grupos de investigación para la si- mulación de datos medidos con muy buenos resultados, mostrando la potencialidad del programa (33) (34) (35) (36).

El programa SIMEDIF para Windows admite hasta 50 zonas térmicas. Para describir un edificio se lo subdivide en locales (zonas que pueden considerarse isotermas). Esta única temperatura evoluciona en el tiempo según lo determinado por el programa, conociendo los datos constructivos y de emplazamiento espacial y temporal del edificio, la variación de temperatura externa en el período a considerar y la radiación solar. Los locales están térmicamente conectados entre sí y con el exterior a través de paredes, tabiques o ventanas.

El modelo admite el ingreso hora a hora de las ganancias internas (disipación metabólica, cocción de alimentos, calefacción auxiliar etc.), aspecto altamente favorable para simular edificios en condiciones reales de uso.

El método de cálculo consiste en un esquema de diferencias finitas explícito avanzando a intervalos de tiempo $\Delta \mathrm{t}$, donde $\Delta$ t es un submúltiplo de una hora. Las temperaturas incógnitas en el problema son las temperaturas de los locales y las temperaturas de los nodos que el esquema en diferencias finitas define en cada elemento con masa (32).

Ganem et al. (30) ha validado el modelo del prototipo de vivienda en SIMEDIF mediante el ajuste de la simulación con datos experimentales. El coeficiente de determinación R2 entre las mediciones y la simulación resultante es del $81 \%$, lo que significa que sólo un $19 \%$ de la variabilidad de los datos no es explicado por el modelo de simulación en SIMEDIF. Por lo tanto, es posible realizar cambios en las condiciones climáticas de sus alrededores, a fin de analizar en forma confiable el impacto sobre la temperatura interior y la demanda de energía auxiliar.

\subsection{Método de monitoreo y recopilación de datos}

Para el desarrollo de las simulaciones, se ha seguido lo explicitado en el Manual de Uso del programa (37). El software necesita los datos meteorológicos externos de temperatura y radiación horarias. Los datos de temperatura se han obtenido a partir del promedio horario de los datos de las estaciones fijas de monitoreo localizadas en cada uno de los casos analizados. En cada uno de los canales analizados, desde mediados de diciembre de 2009 hasta febrero de 2010, se han instalado
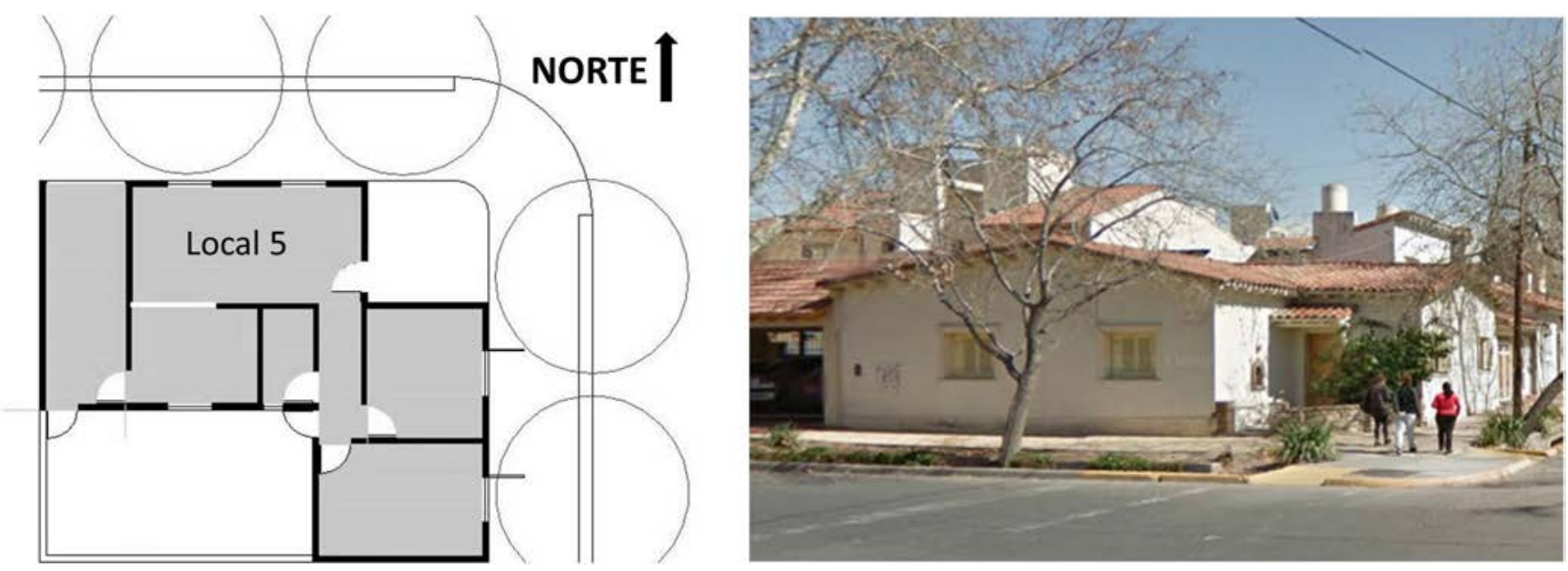

Figura 2. Planta y vista de la vivienda. Fuente: Adaptado de Ganem et al. (30) y Street View (C) 2015 Google Inc. 
nueve estaciones fijas de medición automática. Las estaciones instaladas son del tipo Ho8-003-02 y miden temperatura y humedad cada 15 minutos. Los sensores se han colocado a una altura de 2,5 $\mathrm{m}$ de la calle, dentro de cajas blancas de PVC perforado, para evitar la irradiación y asegurar la circulación adecuada de aire (38). La Tabla 1 muestra las temperaturas exteriores medias, máximas y mínimas en cada caso de estudio durante el periodo evaluado, tanto en verano como en invierno. La radiación global horizontal ha sido tomada de la Estación Meteorológica Pegasus del Centro Científico Tecnológico CCT-Mendoza - CONICET.

El período de simulación de verano estuvo comprendido entre el 16 y el 20 de enero de 2010 caracterizado por días cla- ros. Con el fin de alcanzar las condiciones iniciales para el período de monitoreo se simularon 10 días previos. Para la situación de invierno, se corrieron un total de 20 días, desde el 22 de julio al 10 de agosto de 2010. Para el análisis se seleccionó el período comprendido entre el 28 de julio y el $1^{\circ}$ de agosto, dado que presentaron condiciones de cielo claro. Los días previos se utilizaron para alcanzar las condiciones iniciales.

En la Figura 3 se ubican en el plano de la ciudad de Mendoza los CVU seleccionados y, el CCT-Mendoza. Como se puede observar, los sitios de medición se encuentran dentro de un radio de $4 \mathrm{~km}$. Esta distancia es válida de acuerdo a lo indicado para los registros de radiación solar de la Red Solarimé-

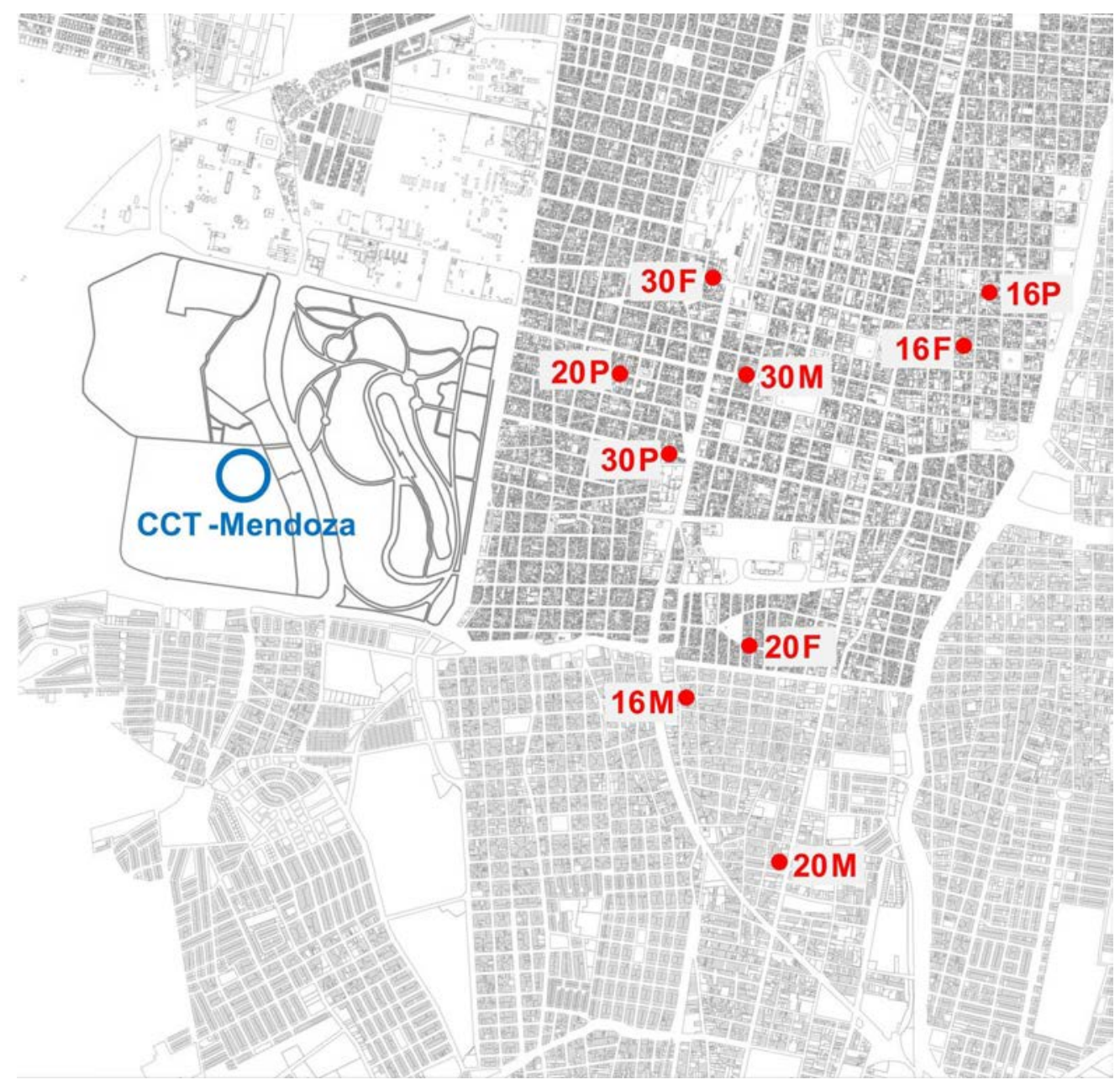

Figura 3. Ubicación de los CVU evaluados (puntos rojos) y del CCT-Mendoza (círculo azul).

Tabla 1. Temperaturas exteriores mínimas, medias y máximas en cada CVU, en invierno y verano.

\begin{tabular}{|l|c|c|c|c|c|c|c|c|}
\hline \multirow{2}{*}{ Especie forestal } & \multirow{2}{*}{$\begin{array}{c}\text { Ancho del } \\
\text { CVU }\end{array}$} & \multirow{2}{*}{ Denominación } & \multicolumn{3}{|c|}{ Invierno } & \multicolumn{3}{c|}{ Verano } \\
\cline { 3 - 9 } & & & Mínima & Media & Máxima & Mínima & Media & Máxima \\
\hline \multirow{3}{*}{ Platanus hispanica } & $16 \mathrm{~m}$ & $16 \mathrm{P}$ & 0.51 & 7.44 & 15.81 & 17.14 & 27.55 & 37.88 \\
\cline { 2 - 9 } & $20 \mathrm{~m}$ & $2 \mathrm{P}$ & -0.95 & 6.84 & 16.38 & 16.10 & 26.84 & 36.68 \\
\cline { 2 - 9 } & $30 \mathrm{~m}$ & $30 \mathrm{P}$ & -0.95 & 7.18 & 16.29 & 16.19 & 27.88 & 39.22 \\
\hline \multirow{3}{*}{ Morus alba } & $16 \mathrm{~m}$ & $16 \mathrm{M}$ & -0.72 & 7.00 & 16.95 & 17.14 & 28.42 & 41.64 \\
\cline { 2 - 9 } & $20 \mathrm{~m}$ & $20 \mathrm{M}$ & -0.27 & 6.93 & 16.57 & 17.14 & 27.87 & 37.33 \\
\hline \multirow{3}{*}{ Fraxinus excelsior } & $30 \mathrm{~m}$ & $30 \mathrm{M}$ & -0.39 & 7.41 & 17.05 & 16.86 & 27.91 & 37.44 \\
\cline { 2 - 9 } & $16 \mathrm{~m}$ & $16 \mathrm{~F}$ & 0.51 & 7.81 & 15.81 & 17.05 & 27.83 & 36.89 \\
\cline { 2 - 9 } & $20 \mathrm{~m}$ & $20 \mathrm{~F}$ & -0.05 & 7.72 & 17.62 & 16.67 & 28.04 & 41.17 \\
\cline { 2 - 9 } & $30 \mathrm{~m}$ & $30 \mathrm{~F}$ & -0.27 & 7.33 & 16.48 & 16.10 & 28.14 & 41.76 \\
\hline
\end{tabular}


trica de la República Argentina por Grossi Gallegos y Lopardo (39). La influencia diferencial de la radiación en cada canal vial urbano se simuló mediante el ingreso de distintas áreas de radiación. El área de las paredes que dan al cañón vial fue afectada por el SVF de cada canal vial. Las superficies de los techos que dan al Norte también han sido modificadas por el SVF en función de la profundidad de la vivienda. En el caso de los techos que dan al Este, se ha realizado el mismo procedimiento con la salvedad de que sólo están sombreados durante las horas de la mañana, por lo que el área sólo ha sido reducida en el 50\%.

El programa también requiere que se ingrese una primera temperatura para comenzar el cálculo, denominada «temperatura inicial de cálculo». Se ha utilizado la temperatura media diaria de cada periodo, es decir del mes de enero y del mes de julio.

Todos los cerramientos se consideraron como muros de ladrillo de la zona, con un coeficiente de conducción de $0,72 \mathrm{~W}$ $\mathrm{m}^{-1}{ }^{\circ} \mathrm{C}^{-1}$, una densidad de $1797 \mathrm{~kg} / \mathrm{m}^{3}$ y una capacidad calorífica de $919 \mathrm{~J} \mathrm{~kg}^{-1}{ }^{\circ} \mathrm{C}^{-1}$. Para los techos se consideró un coeficiente de $0,5 \mathrm{~W} \mathrm{~m}^{-2}{ }^{\circ} \mathrm{C}^{-1}$.

En cuanto a los coeficientes convectivos que se aplican sobre paredes y tabiques, es necesario especificar según el tipo de elemento. Para superficies exteriores, el coeficiente global convectivo-radiativo se ha calculado a partir de la relación Duffie y Beckman (40) que se presenta en la Ecuación [1] donde $\mathrm{v}$ es la velocidad del aire en $\mathrm{m} / \mathrm{s}$.

$$
h=5,7+3,8 . v
$$

En este estudio se han tomado los promedios horarios de $\mathrm{v}$ provenientes de las campañas de medición con estaciones móviles durante enero 2009 y 2010 y se han relacionado con los de la estación Pegasus. Las estaciones móviles marca HOBO $囚$, modelo H21-001 registraron temperatura y humedad relativa del aire, presión atmosférica, velocidad y dirección del viento, radiación solar cada 15 minutos. Para los datos del invierno se ha buscado la misma proporción respecto de los datos observados en la estación Pegasus.

Para las paredes y tabiques internos, los coeficientes de transferencia por convección de este estudio se han tomado los valores de $6 \mathrm{~W} \mathrm{m-2}{ }^{\circ} \mathrm{C}-1$ para aquellas superficies internas que no reciben radiación solar y $8 \mathrm{~W} \mathrm{m-2}{ }^{\circ} \mathrm{C}-1$ para aquellas paredes y tabiques internos que reciben radiación solar.

A partir de la información y la selección de los parámetros discutidos previamente, se procedió con las simulaciones a fin de obtener las temperaturas interiores de la vivienda analizada, inserta en cada uno de los canales viales evaluados. De este modo fue posible conocer la influencia del ambiente exterior sobre el comportamiento térmico de la misma.

Posteriormente se establecieron temperaturas de confort para cada estación y se calculó la energía auxiliar necesaria para mantener la temperatura interior de la vivienda en situación de confort, es decir la energía utilizada para calefacción en invierno o para refrigeración en verano.

Según Givoni (41), los límites de la zona de confort en climas cuya humedad atmosférica absoluta es media o baja resultan independientes del contenido de humedad, ya que el confort de las personas en estado sedentario y con ropa común no se ve afectado por esta variable. Cuando la humedad atmosférica absoluta es alta los efectos de la temperatura y la humedad están interrelacionados y el límite superior de temperatura disminuye a medida que aumenta la humedad.

Los límites de temperatura de confort sugeridos cuando el aire está quieto son de 18 a $25^{\circ} \mathrm{C}$ en invierno y de 20 a $27^{\circ} \mathrm{C}$ en verano. Este límite de temperatura superior es aplicable a bajos niveles de humedad (por debajo de un contenido de vapor de $12 \mathrm{~g} / \mathrm{kg}$ ). A humedades más altas el límite superior de temperatura disminuye progresivamente. Para las personas que viven en países en vías de desarrollo de clima cálido el autor sugiere elevaciones de aproximadamente $2^{\circ} \mathrm{C}$ en los límites de temperatura, teniendo en cuenta la aclimatación como resultado de vivir en edificios sin aire acondicionado en un clima caliente. En invierno, las bajas temperaturas y la situación energética local determinan que los límites de confort no se modifiquen, de manera que los mismos se encuentran entre 18 y $25^{\circ} \mathrm{C}$.

En la ciudad de Mendoza, durante el verano, las condiciones de humedad absoluta habitualmente se sitúan por debajo de los $12 \mathrm{~g} / \mathrm{kg}$. El uso del aire acondicionado no es generalizado, y en particular, la vivienda que se analiza no cuenta con esta tecnología. Sin embargo, el AMM presenta una estación fría y una cálida, bien diferenciadas, con estaciones intermedias de otoño y primavera. Por lo tanto, sólo se aumenta en $2{ }^{\circ} \mathrm{C}$ el límite inferior del rango de confort en el interior de la vivienda para la situación de verano y el rango de confort se define entre los 22 y los $27^{\circ} \mathrm{C}$ para la estación estival.

\section{RESULTADOS}

\subsection{Temperatura interior de la vivienda}

Se consignan aquí los resultados obtenidos en el espacio seleccionado (Local 5), que funciona como estar-comedor, para el inverno y para el verano.

En líneas generales, se puede decir que tanto en invierno como en verano las viviendas más frescas son las ubicadas en los cañones viales forestados con Platanus hispanica. Esto se debe principalmente a las bajas temperaturas exteriores registradas en esos canales y a la escasa radiación solar recibida resultado del bajo factor de visión de cielo de estos cañones urbanos (ver Figura 1 y Tabla 1). Asimismo, se constata que las temperaturas interiores son menos influenciadas por el entorno en invierno que en verano debido a que los forestales evaluados son caducifolios, es decir que no cuentan con hojas que cumplan la función de protección solar durante el invierno, lo que hace que las especies se parezcan entre sí.

El caso del plátano es particular ya que se trata de una especie caducifolia pues entra en reposo con las bajas temperaturas del invierno. Sin embargo, esta especie presenta una particularidad: las hojas muertas quedan sobre el árbol protegiendo las yemas de las nuevas hojas que brotarán en la primavera. Esta característica se denomina como marcescente. Por lo tanto, el SVF no se modifica sustancialmente entre el verano y el invierno, a pesar de que esas hojas no cumplan las funciones de fotosíntesis, respiración ni transpiración.

Durante el invierno, cuando se analizan los canales viales de $16 \mathrm{~m}$ de ancho, se observa que durante la madrugada y hasta las 8:0o hs (horario solar) las viviendas ubicadas en el canal 
forestado con plátanos y en el forestado con moreras muestran similares temperaturas. Sin embargo, durante el resto del día, la temperatura del prototipo edilicio inserto en un canal vial urbano (CVU) forestado con moreras escala hasta asemejarse a la del CVU forestado con fresnos. Este comportamiento puede deberse a que el canal con Morus alba presenta un hueco en el extremo Oeste, por lo que la radiación solar accede en mayor medida que si tuviese un desarrollo homogéneo.

En las viviendas ubicadas en cañones urbanos de $20 \mathrm{~m}$ de ancho se observan las mayores diferencias entre sus temperaturas interiores de acuerdo a la especie con la que se halla forestado. La residencia más fresca es la situada en el CVU con plátanos, le sigue aquella que se encuentra en el CVU forestado con moreras $\left(0,6^{\circ} \mathrm{C}\right.$ más); siendo la vivienda inserta en el canal vial forestado con fresnos la más cálida $\left(0,8^{\circ} \mathrm{C}\right.$ más). La diferencia térmica promedio entre la más fresca y la más cálida es de $1,4^{\circ} \mathrm{C}$.

Las viviendas localizadas en CVU de $30 \mathrm{~m}$ de ancho presentan una situación intermedia entre los dos casos anteriores ya que si bien el comportamiento de la temperatura interior es semejante dado que su orden es el mismo que en los CVU de $20 \mathrm{~m}$, la diferencia de temperatura es menor.

En todas las situaciones evaluadas el prototipo simulado en el cañón urbano de $20 \mathrm{~m}$ de ancho forestado con plátanos es el más fresco de todos en el invierno. Al realizar un análisis de las curvas por especie, vemos que cuando la vivienda se inserta en canales forestados con fresnos presenta las mayores temperaturas interiores de invierno tanto en los canales de $20 \mathrm{~m}$ como de $30 \mathrm{~m}$, que muestran similitud en los valores de la temperatura interior de la vivienda, sin embargo la vivienda situada en el CVU de $16 \mathrm{~m}$ presenta temperaturas interiores de invierno levemente inferiores. En el caso de moreras y plátanos, cuando la vivienda se inserta en los CVU de 16 y $30 \mathrm{~m}$ muestra temperaturas interiores de invierno muy similares, mientras que cuando se inserta en CVU de $20 \mathrm{~m}$ es más fresca. Esto sostiene la hipótesis de que es la combinación entre el acceso a la radiación solar y la condición de abrigo que ofrece un canal vial, el efecto que comanda la temperatura interior de la vivienda. Dado que el canal de 16 m compensa con el encajonamiento (condición de abrigo) su menor acceso a la radiación solar, mientras que el canal de $30 \mathrm{~m}$ logra con un mayor acceso a la radiación solar (para el caso de plátanos y moreras) y tiene mayor exposición para el enfriamiento radiante y convectivo.

En el análisis del verano se puede decir que las viviendas más calurosas son las ubicadas en canales viales de $30 \mathrm{~m}$ de ancho y de éstos, el forestado con fresnos presenta la situación más crítica. Esto se explica principalmente en base del gran factor de visión de cielo que presenta este CVU cuyo valor es de $75 \%$, lo que sumado a la relación entre la forma del espacio y el recorrido solar, aumenta su condición de acceso al sol. Cabe mencionar que las diferencias entre viviendas ubicadas en distintos canales se mantienen relativamente constantes a lo largo del día.

Dentro de los cañones urbanos de $16 \mathrm{~m}$ de ancho, la vivienda a la sombra de moreras es la más cálida con una temperatura máxima media de $31,6^{\circ} \mathrm{C}$. El «hueco» que presenta este canal vial determina una alta proporción de radiación solar que llega tanto a pavimentos vehiculares y peatonales como a las fachadas, donde parte es acumulada y parte es reflejada. Con una diferencia de aproximadamente un grado $\left(0,9^{\circ} \mathrm{C}\right)$, le sigue la edificación a la sombra de fresnos y por último se encuentra aquella sombreada por plátanos con $28,5^{\circ} \mathrm{C}$ de temperatura máxima media. Es decir que existe una diferencia en la temperatura interior de la vivienda superior a $2{ }^{\circ} \mathrm{C}$ entre la localización más favorable y la menos favorable.

En cuanto a los CVU de 20 m de ancho, se observa una diferencia importante de temperatura interior de verano entre la vivienda ubicada en el canal forestado con fresnos y la ubicada en el canal forestado con moreras $\left(1,7^{\circ} \mathrm{C}\right)$. A su vez, el local 5 sombreado con plátanos es en promedio $1^{\circ} \mathrm{C}$ más fresco que el sombreado con moreras de manera que obtiene las mínimas temperaturas observadas con alrededor de $27,2^{\circ} \mathrm{C}$ de temperatura mínima media. Es decir que existe aproximadamente $3{ }^{\circ} \mathrm{C}$ de diferencia en la temperatura interior como consecuencia de la localización más favorable (plátanos) y la más desfavorable (fresnos). Cabe destacar que las temperaturas simuladas bajo las condiciones del CVU de $20 \mathrm{~m}$ de ancho forestado con plátanos en verano son las únicas que entran dentro de los rangos de confort sugeridos por Givoni (41).

Por último, se encuentran las viviendas simuladas bajo las condiciones microclimáticas de los canales viales de $30 \mathrm{~m}$ de ancho. El prototipo ubicado en el CVU forestado con fresnos es el que presenta mayor temperatura interior con máximas de alrededor de $32,4^{\circ} \mathrm{C}$. El que se encuentra en el CVU forestado con moreras es $0,9^{\circ} \mathrm{C}$ más fresco y le sigue el que está a la sombra de plátanos con $0,8^{\circ} \mathrm{C}$ menos en promedio. Es decir que existe una diferencia superior a $1,5^{\circ} \mathrm{C}$ entre la temperatura interior de la vivienda en la localización más favorable y la menos.

Para el caso de verano, nuevamente se concluye que como era de esperar la variable exterior de mayor influencia sobre la temperatura interior es la condición de acceso al sol que cada una de las estructuras evaluadas presenta.

\subsection{Consumo energético en el interior de la vivienda}

Se decidió termostatizar cada local a temperaturas interiores de $20^{\circ} \mathrm{C}$ en invierno y $25^{\circ} \mathrm{C}$ en verano, a fin de observar su efecto en la energía auxiliar de calefacción y refrigeración (41). Los resultados muestran que el consumo de calefacción en invierno está en el orden de los 445-510 MJ/día para la vivienda completa, mientras que la carga diaria de refrigeración en el verano varía entre los 280 y los $400 \mathrm{MJ} /$ día. Esto significa que el invierno es alrededor del $37 \%$ más crítico que la estación estival en el Área Metropolitana de Mendoza, lo cual se condice con resultados obtenidos en trabajos previos (42) (43).

Por otro lado, se puede observar la mayor influencia de la vegetación durante el verano evidenciada por las mayores diferencias entre los SVF y su relación con la trayectoria solar (acceso al sol) de los distintos canales viales estudiados. Este fenómeno es coherente con el hecho de que los forestales evaluados, así como la mayoría de los utilizados en el AMM como arbolado en alineación, son especies caducifolias, por lo que pierden las hojas en la estación invernal ya que entran en reposo vegetativo.

En el análisis de la situación de invierno, se puede ver que la vivienda ubicada en el canal vial de $20 \mathrm{~m}$ de ancho forestado 
con plátanos es la que más energía necesita para mantenerse a $20^{\circ} \mathrm{C}$, mientras que el menor consumo de energía por calefacción está representado por el prototipo simulado en el cañón urbano también de $20 \mathrm{~m}$ de ancho, pero forestado con $\mathrm{F}$. excelsior (12\% menos), especie de menor desarrollo de copa y que por lo tanto, permite un mayor acceso a la radiación solar. Esta situación se condice con las temperaturas interiores que se muestran en el apartado precedente.

La energía necesaria para mantener la temperatura interior en $25^{\circ} \mathrm{C}$ durante el verano es mínima en la casa localizada en el canal vial de $20 \mathrm{~m}$ de ancho forestado con plátanos y máxima en la que se ha simulado en el CVU de $30 \mathrm{~m}$ de ancho forestado con fresnos ( $43 \%$ más).

La Figura 4 consigna los valores de energía auxiliar por m2 de superficie. En verano, la vivienda ubicada en el CVU de $20 \mathrm{~m}$ de ancho forestado con P. hispanica consume $30 \%$ menos de energía de lo que consume la residencia localizada en el canal de $30 \mathrm{~m}$ forestado con $\mathrm{F}$. excelsior. Es decir que en la ciudad de Mendoza, una misma vivienda puede ahorrar hasta 30\% de energía durante el verano debido sólo al ancho del CVU en el que está localizada y la especie forestal utilizada en el mismo.

Cabe destacar que la forestación con moreras resulta en un caso intermedio entre los plátanos y los fresnos ya que se trata de una especie de segunda magnitud al igual que el fresno, pero presenta una mayor superposición de copas a la distancia que habitualmente es plantada en la ciudad de estudio, lo que la asemeja a P. hispanica.

\section{DISCUSIÓN}

Como se ha dicho precedentemente, el caso que permite el mayor ahorro energético durante el verano es el que causa el mayor gasto de energía en el invierno. Este hecho reconoce la gran importancia del recurso solar en ciudades de zonas áridas como el AMM, el que es determinante de la mayor parte de los procesos de intercambio de calor que se producen en los espacios abiertos e interfieren, como se manifiesta en este trabajo, con el desempeño térmico de los espacios interiores y el consumo de energía del parque edilicio.

Por otro lado, es necesario destacar que el consumo energético de la vivienda puede ser disminuido mediante interven- ciones edilicias (utilización de pinturas reflectivas, aislación térmica, etc.), las cuales tienden a generar mayores beneficios con un costo también más alto (44) (45).

Sin embargo, cuando las posibilidades de acondicionamiento interior se ven reducidas y se desea minimizar los consumos energéticos mediante el acondicionamiento del espacio urbano y la adecuada elección de la vegetación, es claro que los mismos deben ser establecidos, según lo evaluado, en la estación estival ya que es en la que se observan las influencias más importantes del verde urbano en los consumos energéticos residenciales.

Se debe tener en cuenta, además, que esta ciudad está emplazada en una zona árida y cuenta ya con una trama forestal consolidada, de la cual depende para garantizar la habitabilidad térmica de los espacios exteriores, es decir sus condiciones de confort (Ruiz et al, 2015). Además, es demandante mitigar la isla de calor presente en la ciudad para mejorar tres aspectos principalmente: confort térmico exterior, posibilidad de ventilación para asegurar una adecuada calidad del aire y enfriamiento nocturno para minimizar consumos energéticos.

En este sentido, se presentan dos situaciones. Por un lado, la renovación de la forestación urbana. El trabajo de Cantón et al (22) revela la condición de una arboleda envejecida y una reducida renovación de la población vegetal, con un $73 \%$ de población forestal madura, siendo la población joven baja (16\%) y la población nueva escasa (2\%). En las zonas más antiguas y céntricas de la ciudad, donde el tránsito es intenso durante gran parte del día, es importante privilegiar las condiciones de ventilación para favorecer la purificación del aire. Esto se puede lograr reforestando con Morus alba los canales de 20 y $30 \mathrm{~m}$ de ancho y con Fraxinus excelsior los de 16 $\mathrm{m}$. Por otro lado, las nuevas urbanizaciones. En los sectores donde se extiende la ciudad se podrá minimizar los consumos energéticos forestando con Platanus hispánica o alguna otra especie de primera magnitud.

Asimismo, la vegetación urbana cumple numerosos servicios ambientales y sociales que contribuyen a aumentar la calidad de la vida en las ciudades. Además de regular el clima (46), proveer sombra (47) y mitigar el efecto de isla de calor (48); la vegetación urbana elimina la contaminación atmosférica (49) (50), colabora en el manejo de aguas plu-

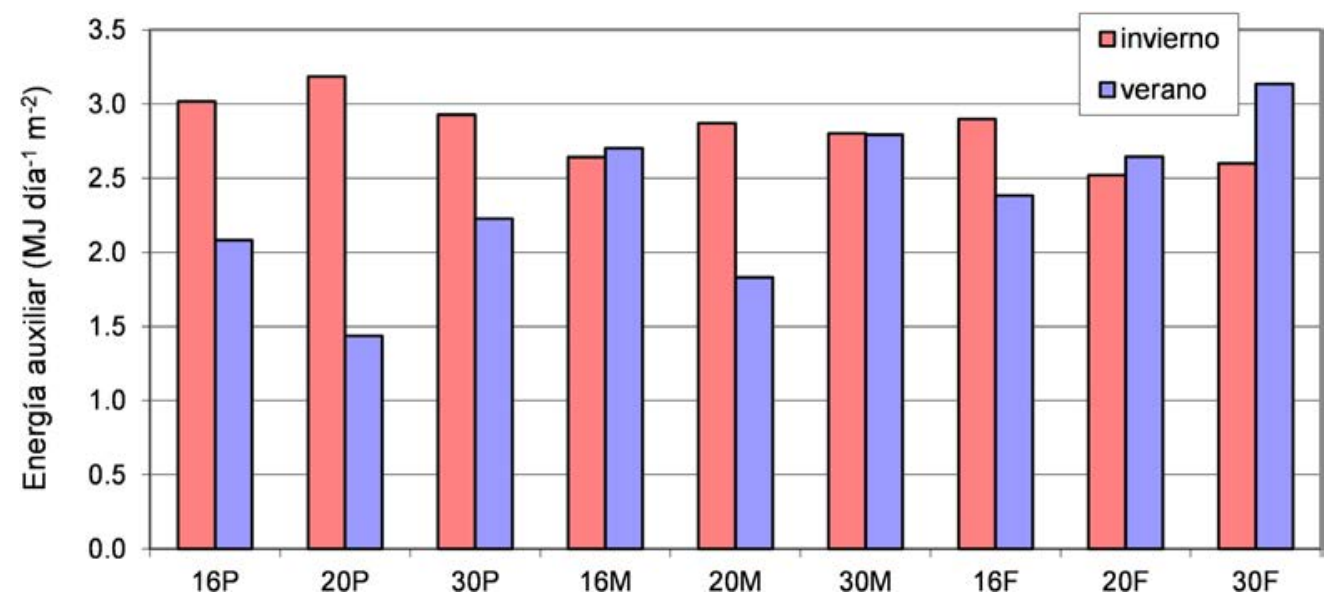

Figura 4. Consumo de energía diario por metro cuadrado para la vivienda estudiada en cada uno de los CVU seleccionados, en invierno y verano. 
viales (51), mejora la salud de los ciudadanos (52) y agrega valor estético a la ciudad (53). Por lo tanto, es importante también incorporar en el diseño de la ciudad vegetación en otro tipo de formatos, desde los más tradicionales (plazas, parques) hasta los más novedosos (techos y paredes verdes). Los mismos también deben ser estudiados en profundidad ya que cada uno tiene características propias que los pueden hacer más o menos eficientes, sobre todo teniendo en cuenta que se trata de una zona en la que el recurso hídrico es escaso. También pueden encontrarse estudios en estas áreas para el caso del AMM, como por ejemplo los trabajos de Correa et al. (54), Ruiz et al. (15), Stocco et al. (55) y Flores Azin et al. (56).

No obstante, la vegetación urbana no debe ser vista como una panacea sino que debe articularse y complementarse con otras herramientas del diseño urbano. En este sentido, Ewing \& Rong (57) vincularon la forma urbana con el uso de energía residencial y encontraron que los residentes de las ciudades expansivas de EEUU son más propensos a vivir en casas unifamiliares aisladas que los residentes de otras ciudades comparables y también más propensos a vivir en casas grandes. Ambos conducen a un mayor uso de energía residencial. En Singapur, Wong et al. (58), identificaron y evaluaron un total de 32 casos, considerando diferentes morfologías urbanas, para dar una mejor comprensión de la implicación de las formas urbanas. Los resultados indicaron un ahorro energético de $4,5 \%$ si se abordan los elementos urbanos eficazmente.

\section{CONCLUSIONES}

El presente trabajo ha evaluado el efecto de diferentes combinaciones de arbolado en alineación y perfil urbano sobre el consumo de energía residencial. Para esto se han simulado las temperaturas interiores y los consumos energéticos necesarios para alcanzar el confort interior de un prototipo repre- sentativo de vivienda localizada en la zona de baja densidad edilicia del Área Metropolitana de Mendoza, tanto en invierno como en verano.

Los resultados apoyan la importancia de la combinación entre el ancho del canal vial y la especie forestal sobre el desempeño termo-energético interior de la edilicia colindante. Durante el verano, esta conjugación entre dos factores de diseño urbano puede producir hasta $3{ }^{\circ} \mathrm{C}$ de diferencia de temperatura interior y hasta $30 \%$ de ahorro energético entre el diseño urbano más favorable y el más desfavorable.

Los resultados brindan parámetros que pueden ser de utilidad en la confección de futuros planes de forestación o reforestación de las ciudades de zonas áridas. En este sentido, se presentan dos situaciones: a) renovación de la forestación urbana y b) nuevas urbanizaciones. En el primer caso, es importante privilegiar las condiciones de ventilación para favorecer la purificación del aire mediante la reforestación de los canales de 20 y $30 \mathrm{~m}$ de ancho con Morus alba y, los de 16 $\mathrm{m}$ con Fraxinus excelsior. En el segundo caso, se podrá minimizar los consumos energéticos forestando con Platanus hispánica.

Además, se han aportado criterios susceptibles de ser incorporados en los códigos urbanos a fin de vincular la fisonomía forestal con la morfología urbana, sobre todo en «ciudades oasis» donde el rol de la forestación es clave para garantizar la sustentabilidad del desarrollo urbano.

\section{AGRADECIMIENTOS}

Este trabajo ha sido financiado por la Agencia Nacional de Promoción Científica y Tecnológica (ANPCYT) mediante del proyecto PICT 2011-0611; así como también por el Consejo Nacional de Investigaciones Científicas y Técnicas (CONICET), PIP 2011-00640.

\section{REFERENCIAS}

(1) Oke, T.R. (1973). City size and the urban heat island. Atmospheric Environment, 7: 769-779, doi: http://dx.doi. org/10.1016/0004-6981(73)90140-6.

(2) Rizwan, A.M., Dennis, Y.C.L., Liu, C. (2008). A review on the generation, determination and mitigation of Urban Heat Island. Journal of Environmental Sciences, 20: 120-128, doi: http://dx.doi.org/10.1016/S1001-0742(08)60019-4.

(3) Cui, L., Shi, J. (2012). Urbanization and its environmental effects in Shanghai, China. Urban Climate, 2: 1-15, doi: http:// dx.doi.org/10.1016/j.uclim.2012.10.008.

(4) Santamouris, M., Papanikolaou, N., Livada, I., Koronakis, I., Georgakis, C., Argiriou, A., Assimakopoulos, D.N. (2001). On the impact of urban climate on the energy consumption of buildings. Solar Energy, 70(3): 201-216, doi: http://dx.doi. org/10.1016/Soo38-092X(oo)00095-5.

(5) Giannakopoulos, C., Psiloglou, B.E. (2006). Trends in energy load demand for Athens, Greece: weather and non-weather related factors. Climate Research, 31: 97-108, doi: http://dx.doi.org/10.3354/cro31097.

(6) Pérez-Lombard, L., Ortiz, J., Pout, C. (2008). A review on buildings energy consumption information. Energy and Buildings, 40: 394-398, doi: http://dx.doi.org/10.1016/j.enbuild.2007.03.007.

(7) Akbari, H., Davis, S., Dorsano, S., Huang, J., Winert, S. (1992). Cooling our Communities. A Guidebook on Tree Planting and Light-Colored Surfacing, Washington D.C.: US Environmental Protection Agency, Office of Policy Analysis, Climate Change Division.

(8) INDEC (2012). Publicación del Censo Nacional de Población, Hogares y Viviendas 2010. Censo del Bicentenario. Resultados definitivos. Serie B No 2, Buenos Aires: Instituto Nacional de Estadísticas y Censos.

(9) Correa, E.N. (2006). Isla de Calor Urbana. El Caso del Área Metropolitana de Mendoza (Tesis doctoral no publicada). Salta, Argentina: Facultad de Ciencias Exactas, Universidad Nacional de Salta.

(10) Robinette, G.O. (1972). Plants, People and Environmental Quality. A Study of Environmental Functions, Washington D.C.: U.S Department of Interior, National Park Service. 
(11) Yilmaz, S., Toy, S., Yilmaz, H. (2007). Human thermal comfort over three different land surfaces during summer in the city of Erzurum, Turkey. Atmósfera, 20(3), 289-297. Recuperado en 14 de septiembre de 2016, de http://www.scielo. org.mx/scielo.php?script=sci_arttext\&pid=S0187-62362007000300004\&lng=es\&tlng=.

(12) Morakinyo, T.E., Balogun, A.A., Adegun, O.B. (2013). Comparing the effect of trees on thermal conditions of two typical urban buildings. Urban Climate, 3: 76-93, doi: http://dx.doi.org/10.1016/j.uclim.2013.04.002.

(13) Correa, E., Ruiz, M.A. Cantón, M.A., Lesino, G. (2012). Thermal comfort in forested urban canyons of low building density. An assessment for the city of Mendoza, Argentina. Building and Environment, 58: 219-230, doi: http://dx.doi. org/10.1016/j.buildenv.2012.06.007.

(14) Puliafito, S.E., Bochaca, F., Allende, D. (2013). Mitigación de la isla de calor urbana en ciudades de zonas áridas. Proyecciones, 11(2).

(15) Ruiz, M.A., Correa, E.N. (2014). Developing a Thermal Comfort Index for Vegetated Open Spaces in Cities of Arid Zones. Energy Procedia, 57: 3130-3139, doi: http://dx.doi.org/10.1016/j.egypro.2015.06.056.

(16) Ruiz, M.A., Correa Cantaloube, E.N., Cantón, M.A. (2015). Incidencia de la selección de la especie forestal en el confort térmico de cañones urbanos de zonas áridas: el caso de Mendoza, Argentina. Revista Urbano, 32: 60-69.

(17) Kottek, M., Grieser, J., Beck, C., Rudolf, B., Rubel, F. (2006). World map of the Köppen-Geiger climate classification updated. Meteorologische Zeitschrift, 15(3): 259-263, doi: http://dx.doi.org/10.1127/0941-2948/2006/0130.

(18) González Loyarte, M.M., Menenti, M., Diblasi, A.M. (2009). Mapa bioclimático para las Travesías de Mendoza (Argentina) basado en la fenología foliar. Revista de la Facultad de Ciencias Agrarias - UNCuyo, XLI(1): 105-122. Recuperado de http://www.redalyc.org/articulo.oa?id=382837644006.

(19) Bórmida, E. (1986). Mendoza, una ciudad oasis. Summa, 226: 68-72.

(20) McPherson, E.G. (1988). Functions of Buffer Plantings in Urban Environments. Agriculture, Ecosystems and Environment, 22: 281-298.

(21) Álvarez, A. (2000). La redefinición territorial del Área Metropolitana de Mendoza en el contexto de los actuales procesos de transformación. Revista GeoNotas 4(4). Recuperado en 10 de octubre de 2015, de http://www.geonotas.uem.br/ geonotas/

(22) Cantón, M.A., De Rosa, C., Kasperidus, H. (2003). Sustentabilidad del bosque urbano en el Área Metropolitana de la ciudad de Mendoza: análisis y diagnóstico de la condición de las arboledas. Avances en Energías Renovables y Medio Ambiente, 7(1): 29-34. https://www.mendoza-conicet.gob.ar/asades/modulos/averma/trabajos/2003/2003-too1-aoo6.pdf.

(23) Correa, E., Martinez, C.F., Cantón, M.A. (2008). Influencia del uso de distintas magnitudes forestales sobre el comportamiento térmico de los cañones urbanos. El caso de la primera magnitud en ciudades de zonas áridas. Avances en Energías Renovables y Medio Ambiente, 12(1): 155-162.

(24) Correa, E., Ruiz, M.A., Cantón, M.A. (2010, 1-5 de octubre). Estrategias de mitigación de la isla de calor urbana. Impacto sobre las condiciones de confort de los espacios abiertos en ciudades de clima semidesértico. En XXXVI Semana Nacional de Energía Solar. Guanajuato, México: Asociación Nacional de Energía Solar.

(25) Ruiz, M.A. (2013). Efectos microclimáticos de la vegetación en ciudades de zonas áridas. Incidencia sobre los consumos energéticos y la calidad ambiental del hábitat (Tesis doctoral no publicada). Salta, Argentina: Facultad de Ciencias Exactas, Universidad Nacional de Salta.

(26) Sosa, M.B., Correa Cantaloube, E., Cantón, M.A. (2016, 23-27 de mayo). ¿En qué medida la forma urbana afecta al comportamiento térmico exterior? Un estudio para reducir la isla de calor urbana en una ciudad árida. En I Encuentro Nacional sobre Ciudad, Arquitectura y Construcción Sustentable. La Plata, Argentina: Facultad de Arquitectura y Urbanismo. Acta del I ENCACS, 91-102.

(27) Arboit, M., de Rosa, C. (2012). Morfología urbana para la sostenibilidad energético ambiental del parque edilicio en ciudades andinas. Caso: Área Metropolitana de Mendoza, Argentina. Avances en Energías Renovables y Medio Ambiente, 16(1): 73-80.

(28) Mesa, N.A. (2003). Método teórico de diagnóstico de la habitabilidad termo-lumínica del espacio arquitectónico, como base para la planificación urbana: caso Mendoza, Argentina. (Tesis doctoral no publicada). Mendoza, Argentina: Doctorado en Arquitectura, Universidad de Mendoza.

(29) Alchapar, N.L., Correa, E.N. (2016). Pautas de diseño para disminuir las temperaturas urbanas en regiones con alta productividad solar. Parámetros morfológicos y materiales. Revista Hábitat Sustentable, 6(1): 84-95.

(30) Ganem, C., Esteves, A., Coch, H. (2005). El rol de la envolvente en la rehabilitación ambiental. Propuestas de Verano para viviendas tipo Chalet en Mendoza. Avances en Energías Renovables y Medio Ambiente, 9(5): 49-54.

(31) García-Alvarado, R., González, A., Bustamante, W., Bobadilla, A., Muñoz, C. (2014). Características relevantes de la simulación energética de viviendas unifamiliares. Informes de la Construcción, 66(533): eoo5 doi: http://dx.doi. org/10.3989/ic.12.108.

(32) Flores Larsen, S., Lesino, G. (2000). SIMEDIF 2000: nueva versión del programa de diseño y cálculo de edificios. Avances en Energías Renovables y Medio Ambiente, 4(8): 53-58. https://www.mendoza-conicet.gob.ar/asades/modulos/ averma/trabajos/2000/2000-too8-a010.pdf.

(33) Hernandez, A., Lesino, G. (2000). Simulación mediante SIMEDIF del comportamiento térmico de un prototipo de vivienda liviana construido en la Universidad Nacional de Salta. Avances en Energías Renovables y Medio Ambiente, 4(8): 29-34.

(34) Filippin, C. (200o). Residencias universitarias solares en la provincia de La Pampa. Su comportamiento higrotérmico y energético-ambiental (Tesis doctoral no publicada). Salta, Argentina: Facultad de Ciencias Exactas, Universidad Nacional de Salta.

(35) Flores Larsen, S., Filippin, C., Lesino, G. (2001). Simulación mediante SIMEDIF y Energy-10 de un edificio liviano. Avances en Energías Renovables y Medio Ambiente, 5(8): 25-30. 
(36) Flores Larsen, S., Filippin C., Lesino G. (2004, 18-21 de julio). Monitoreo y simulación higrotérmica de verano de un conjunto de residencias estudiantiles bioclimáticas. En X Encontro Nacional de Tecnologia do Ambiente Construído (ENTAC 2004) e I Conferência Latinoamericana de Construção Sustentável (claCS 04). San Pablo: Associação Nacional de Tecnologia no Ambiente Construído.

(37) Flores Larsen, S. (2016). SIMEDIF Software for the transient thermal calculation of buildings. Users'manual, p. 30, Salta, Argentina: INENCO - Universidad Nacional de Salta. Recuperado en 5 Marzo de 2018, de http://170.210.201.130/?wpfb_ $\mathrm{dl}=7$.

(38) Oke, T.R. (2004). Initial guidance to obtain representative meteorological observations at urban sites. IOM Report No. 81, WMO/TD No. 1250, Ginebra: World Meteorological Organization.

(39) Grossi Gallegos, H., Lopardo, R. (1988). Spatial variability of the global solar radiation obtained by the Solarimetric Network in the Argentine Pampa Húmeda. Solar Energy, 40: 397-404, doi: http://dx.doi.org/10.1016/oo38092X(88)90094-1.

(40) Duffie, J.A., Beckman, W.A. (1991). Solar Engineering of Thermal Processes, p. 54-59, New York: Wiley-Interscience.

(41) Givoni, B. (1998). Climate considerations in building and urban design, p. 38-39, New York: John Wiley \& Sons, Inc.

(42) Barea, G., Ganem, C., Esteves, A. (2009). Análisis térmico invernal de una vivienda unifamiliar no-tradicional en la Ciudad de Mendoza. Avances en Energías Renovables y Medio Ambiente, 13(5): 195-202.

(43) Mercado, M.V., Esteves, A., Filippin, C. (2010). Comportamiento térmico-energético de una vivienda social de la ciudad de Mendoza, Argentina. Ambiente Construido, 10: 87-101. http://www.scielo.br/pdf/ac/v1on2/ao6.pdf.

(44) Filippin, C., Flores Larsen, S. (2005). Comportamiento térmico de invierno de una vivienda convencional en condiciones reales de uso. Avances en Energías Renovables y Medio Ambiente, 9(5): 67-72.

(45) Filippin, C., Flores Larsen, S. (2006). Comportamiento energético de verano de una vivienda convencional en la región central de Argentina. Avances en Energías Renovables y Medio Ambiente, 10(5): 9-14.

(46) Shashua-Bar, L., Potcher, O., Bitan, A., Boltansky, D., Yaakov, Y. (2009). Microclimate modelling of street tree species effects within the varied urban morphology in the Mediterranean city of Tel Aviv, Israel. International Journal of Climatology. 30(1): 44-57.

(47) Tsiros, I.X. (2009). Assessment and energy implications of street air temperature cooling by shadetress in Athens (Greece) under extremely hot weather conditions. Renewable Energy, 35(8): 1866-1869.

(48) Mayer, H., Kuppe, S., Holst, J., Imbery, F., Matzarakis, A. (2009). Human thermal comfort below the canopy of street trees on a typical Central European summer day. Meteorological Institute, Albert-Ludwigs- University, Freiburg.

(49) Chen, W.Y., Jim, C.Y. (2005). Assessing the ecosystem service of air pollutant removal by urban trees in Guangzhou (China). Journal of Environmental Management, 88(4): 665-676.

(50) Buccolieri, R., Gromke, C., Silvana, S., Ruck, B. (2009). Aerodynamic effects of trees on pollutant concentration in street canyons. Science of the Total Environment, 407(19): 5247-5256.

(51) Clayden, A., Jorgesen, A., Stovin, R. (2008). Street Trees and Stormwater Management. The International Journal of Forestry, 30(4): 297-310.

(52) Lovasi, G.S., Quinn, J.W., Neckerman, K.M., Perzanowski, M.S., Rundle, A. (2008). Children living in areas with more street trees have lower prevalence of asthma. Journal of Epidemiology and Community Health, 62:647-649.

(53) Price, C. (2003). Quantifying the aesthetic benefits of urban forestry. Urban For Urban Green, 1(3):123-133.

(54) Correa, E., Martínez, C., Lesino, G., de Rosa, C., Cantón, A. (2006, 6-8 de septiembre). Impact of Urban Parks on the Climatic Pattern of Mendoza's Metropolitan Area, in Argentina. En: PLEA 2006, 23rd Conference on Passive and Low Energy Architecture. Geneva, Switzerland.

(55) Stocco, S., Cantón, M.A., Correa, E.N. (2015). Design of urban green square in dry areas: Thermal performance and comfort. Urban Forestry \& Urban Greening, 14(2): 323-335, doi: http://dx.doi.org/10.1016/j.ufug.2015.03.001

(56) Flores Asin, J.E., Martinez, C.F., Cantón, M.A., Correa, E.N. (2016, 15-17 de febrero). Comportamiento térmico de cubiertas vegetadas como estrategia para aumentar la sustentabilidad de ciudades en zonas árida. En Congreso Internacional Towards Green Cities. Mérida, México.

(57) Ewing Reid, Rong Fang. 2008. The Impact of Urban form on US Residential Energy Use. Housing Policy Debate, 19(1): 1-30.

(58) Wong, N.H., Jusuf, S.K., Syafii, N.I., Chen, Y., Hajadi, N., Sathyanarayanan, H., Manickavasagam, Y.V. (2011). Evaluation of the impact of the surrounding urban morphology on building energy consumption. Solar Energy, 85: 57-71, doi: http://dx.doi.org/10.1016/j.solener.2010.11.002 